\title{
Neonatal mortality in East Africa and West Africa: a geographic analysis of district-level demographic and health survey data
}

\author{
Sue C. Grady, ${ }^{1,2}$ April N. Frake, ${ }^{3}$ Qiong Zhang, ${ }^{2}$ Matlhogonolo Bene, ${ }^{2}$ Demetrice R. Jordan, ${ }^{3}$ Joshua \\ Vertalka, ${ }^{1}$ Thania C. Dossantos, ${ }^{2}$ Ameen Kadhim, ${ }^{1}$ Judith Namanya, ${ }^{1}$ \\ Lisa-Marie Pierre, ${ }^{1}$ Yi Fan, ${ }^{1}$ Peiling Zhou, ${ }^{2}$ Fatoumata B. Barry, ${ }^{2}$ Libbey Kutch ${ }^{2}$ \\ ${ }^{1}$ Department of Geography, Environment and Spatial Sciences, Michigan State University, East Lansing, \\ MI; ${ }^{2}$ Global Health and Medical Geography Lab, Department of Geography, Environment and Spatial \\ Sciences, Michigan State University, Lansing, MI; ${ }^{3}$ Center for Global Change and Earth Observation, \\ Michigan State University, East Lansing, MI, USA
}

\begin{abstract}
Under-five child mortality declined $47 \%$ since 2000 following the implementation of the United Nation's (UN) Millennium Development Goals. To further reduce under-five child mortality, the UN's Sustainable Development Goals (SDGs) will focus on interventions to address neonatal mortality, a major contributor of under-five mortality. The African region has the highest neonatal mortality rate (28.0 per 1000 live births), followed by that of the Eastern Mediterranean (26.6) and South-East Asia (24.3). This study used the Demographic and Health Survey Birth Recode data (http://dhsprogram.com/data/File-Types-and-Names.cfm) to identify high-risk districts and countries for neonatal mortality in two sub-regions of Africa - East Africa and West Africa. Geographically weighted Poisson regression models were estimated to capture the spatially varying relationships between neonatal
\end{abstract}

Correspondence: Sue C. Grady, Department of Geography, Environment and Spatial Sciences, 673 Auditorium Rd., Rm. 207. Michigan State University, East Lansing, 48824 MI, USA.

Tel: +1.517.432.9998 - Fax: +1.517.432.1671.

E-mail: gradys@msu.edu

Key words: Neonatal mortality; Sustainable development goals; Geographically weighted Poisson regression; Sub-Saharan Africa.

Acknowledgements: the authors of this study were inspired by readings in Geographies of Health and Development, Eds. Isaac Luginaah and Rachel Bezner Kerr. 2015. New York: Routledge Publishing. The authors would also like to thank the anonymous reviewers for their insightful comments.

Received for publication: 15 August 2016.

Revision received: 20 March 2017

Accepted for publication: 22 March 2017.

(C) Copyright S.C. Grady et al., 2017

Licensee PAGEPress, Italy

Geospatial Health 2017; 12:501

doi:10.4081/gh.2017.501

This article is distributed under the terms of the Creative Commons Attribution Noncommercial License (CC BY-NC 4.0) which permits any noncommercial use, distribution, and reproduction in any medium, provided the original author(s) and source are credited. mortality and dimensions of potential need i) care around the time of delivery, ii) maternal education, and iii) women's empowerment. In East Africa, neonatal mortality was significantly associated with home births, mothers without an education and mothers whose husbands decided on contraceptive practices, controlling for rural residency. In West Africa, neonatal mortality was also significantly associated with home births, mothers with a primary education and mothers who did not want or plan their last child. Importantly, neonatal mortality associated with home deliveries were explained by maternal exposure to unprotected water sources in East Africa and older maternal age and female sex of infants in West Africa. Future SDG-interventions may target these dimensions of need in priority high-risk districts and countries, to further reduce the burden of neonatal mortality in Africa.

\section{Introduction}

The year 2015 marked the conclusion of the United Nation's (UN) Millennium Development Goals (MDG) to create a more social, economic, equitable, and sustainable world. Substantial progress was made after September 2000 when world leaders signed the Millennium Declaration to implement eight MDG goals, most notably the global reduction in poverty, improvements in education completion rates, improved access to drinking water and sanitation, decreases in HIV, tuberculosis and malaria prevalence and a reduction in the maternal mortality ratio (UN, 2015). The eight development goals under the MDG will now transition to $17 \mathrm{UN}$ Sustainable Development Goals (SDGs) to be implemented through target date 2030 (UN, 2016).

This study focuses on the transition from MDG 4 to SDG 3.2 to further reduce under-five child mortality. Under the implementation of MDG 4, there was a 53\% reduction in under-five child mortality - from an estimated 90 to 43 deaths per 1000 live births (1990-2015) - equating to the survival of approximately six million children worldwide (UN, 2015). Yet, despite this extraordinary success, approximately 5.9 million children (16,000 children per day) died in 2015. In this cohort of child deaths, $45 \%$ were infants who died during the neonatal period (first 28 days of life) (UN, 2015). Of these neonatal deaths, 36\% occurred in the first 24 hours after birth (early neonatal deaths) (UN, 2015). The neonatal mortality rate was highest in the World Health Organization (WHO) Regions of Africa (28.0), Eastern Mediterranean (26.6), South-East Asia (24.3) and with substantially lower neonatal mortality rates in the Americas (7.7), Western Pacific (6.7) and Europe 
(6.0) (WHO, 2015a). Since 1990, the regions with the greatest percentage reduction in the rate of neonatal mortality were Western Pacific (-75.4\%), Europe (-59.1), Americas (-56.0\%), South-East Asia (-53.9), Eastern Mediterranean (-37.5) and Africa (-34.1\%) (WHO, 2015a). These findings show that regions with the highest neonatal mortality rates (Africa, Eastern Mediterranean and SouthEast Asia) also had smaller reductions in those rates over the last two decades, despite the substantial progress made to reduce under-five child mortality worldwide. Furthermore, according to the United Nations International Children's Fund (UNICEF), neonatal deaths as a percentage of the under-five mortality in 2015 were highest in South-East Asia (57.4\%), Europe (53.0\%), Americas (52.4\%), Eastern Mediterranean (51.4\%), Western Pacific (49.6\%) and Africa (34.9\%) ((UNICEF, 2015); however, since 1990, those regions with the greatest percentage increase in the neonatal death contribution to under-five mortality were Africa (35.7\%), South-East Asia (28.6\%), Americas (27.8\%), Eastern Mediterranean (21.2\%) and Europe (15.7\%). Neonatal mortality as a percentage of the under-five mortality rates increased in all regions except Western Pacific (-7.6\%) (UNICEF, 2015).

In summary, the WHO African Region has the highest neonatal mortality rate and the smallest percent reduction in neonatal mortality since 1990. In Africa, over one-third of under-five child mortality occurred in the neonatal period and that percentage is rising. To that end, targeting future research, policy, and programme interventions to reduce neonatal mortality is a critical step particularly in the WHO Region of Africa.

The major causes of neonatal mortality worldwide are premature birth (births $<37$ weeks gestation) and low-birth weight (infants $<2500 \mathrm{~g})(30 \%)$, neonatal infections (25\%), birth asphyxia (failure to establish breathing at birth) (Lawn et al., 2007) and birth trauma $(23 \%)$, other neonatal causes $(9 \%)$, congenital anomalies (7\%), neonatal tetanus (3\%) and diarrheal diseases (3\%) (WHO, 2004). Engmann et al. (2012) used verbal autopsy to identify the most common causes of death in neonates for mothers living in low-income countries and rural areas and found them to be infectious (45\%), birth/intrapartum asphyxia (lack of oxygen in-utero leading to carbon dioxide retention and metabolic acidosis) $(26 \%)$, preterm birth $(17 \%)$, tetanus (4\%), congenital malformations $(3 \%)$ and other causes $(2 \%)$. In Africa (2004) neonatal deaths $(n=1.16$ million) were largely attributed to infections (38\%) including, sepsis/pneumonia (28\%), tetanus $(6 \%)$ and diarrhoea (4\%), followed by preterm birth $(25 \%)$, asphyxia $(24 \%)$, congenital defects $(6 \%)$ and other causes (7\%) (Lawn et al., 2008). Among this cohort, neonatal deaths caused by birth asphyxia occurred on the first day of life (day 1); sepsis/pneumonia, complications of preterm birth, severe congenital anomalies and other causes generally occurred during the first week of life (days 1-7); neonatal tetanus peaked during days 4-9 of life; and neonatal death from diarrhoea typically occurred toward the end of the first month of life (Lawn et al., 2008). Thus, in low-income countries in general, and in African countries in particular, the major causes of neonatal mortality were birth asphyxia and birth injury, infectious causes and preterm birth. Birth asphyxia and birth injury occur when a newborn fails to breathe spontaneously after delivery or is in shock due to hypovolemia (low blood volume due to dehydration or bleeding from injury). In high-risk pregnancies, approximately $70 \%$ of neonates will be at-risk of asphyxia either because of small body size, poorly developed lungs and respiratory system, pulmonary dysfunction and/or complications in-utero during labour or at the time of delivery. Asphyxia produces hypoxemia (low oxygen) and hypercapnia (carbon dioxide retention), which cause death without rapid resuscitation (Merck Manual, 2015). Skilled birth attendants who assist during delivery and have experience in emergency obstetric care can help to prevent birth asphyxia on day 1 of life, reducing neonatal mortality. Infectious causes of neonatal morality include neonatal sepsis (invasive bacterial infection), pneumonia (aspiration of infected amniotic fluid with prolonged labor), meningitis (bacterial infection of the meninges - usually secondary to neonatal sepsis) and tetanus (neurotoxic bacteria found in the newborn's umbilicus). These infections are transmitted from the mother to infant during childbirth complications or shortly thereafter (days 1-9), and are largely preventable through the presence of a skilled birth attendant at delivery to ensure hygienic childbirth care, including the care of the umbilical cord and the application of the tetanus toxoid vaccine. Other preventative measures to reduce neonatal infection during childbirth and the neonatal period include high quality education for mothers during prenatal, antenatal and postnatal care visits, a clean water supply and early breastfeeding practices to improve infant immunity. Preterm birth (premature labour) may be associated with vaginal bleeding or premature rupture of the amniotic membranes. Infant complications associated with preterm birth may be avoided during days 1-7 with the presence of a trained birth attendant at delivery to identify and treat birth asphyxia and injury and a neonatal intensive care unit to provide the infant with warmth, feeding, hygiene and the early identification of infections and other complications and illnesses (Lawn et al., 2008). Preterm birth itself, may be prevented through the lengthening of gestation with bed-rest and appropriate medical care, maternal education and prenatal and antenatal care, in particular, the identification and treatment of sexually transmitted infections (STIs), human immunodeficiency virus (HIV) and malaria (Lawn et al., 2008).

The SDG to reduce neonatal and under-five mortality now falls under SDG 3 Ensure healthy lives and promote well-being for all at all ages and SDG 3.2 By 2030, end preventable deaths of newborns and children under five years of age - with all countries aiming to reduce neonatal mortality to at least as low as 12 per 1,000 live births and under-five mortality to at least as low as 25 per 1,000 live births (WHO, 2015b). Importantly, the interventions addressing neonatal mortality in the SDG 3.2 (UN, 2016) also address similar interventions relating to maternal mortality, including enhancing the availability and quality of health care services, increase the number of trained health care workers and available medicine and supplies to improve care around the time of delivery and the first week of newborn's life (days 1-7); providing timely preventative care and treatment, including immunisations, vitamin A supplementation and available medical services for the treatments of early and late-onset pneumonia, diarrhoea and malaria (days 1-30); and reducing malnutrition in all forms beginning with early breastfeeding. Finally, improving health system structures such as surveillance of births and infant deaths and managing hospitals and health care centres within conflict areas (UN, 2016) are very important but challenging interventions to reduce neonatal mortality in Africa.

In addition to health and health care interventions to reduce neonatal mortality, SDGs 4 and 5 focus on education and gender equality and rights, which will directly and indirectly impact maternal and neonatal health. Educating mothers is one of the strongest determinants of child survival (Caldwell, 1979; Hobcraft, 1993; Bicego and Boerma, 1993; WHO, 2015b). Primary education teaches women to read, write and work with numbers, increas- 
ing their ability to make informed decisions in regards to healthy nutrition, health-promoting behaviours and the management of money to purchase basic needs. Secondary and tertiary education further increases women's opportunities in the workforce, increasing their ability to access health care (WHO, 2015b) for the promotion of pre-pregnancy and pregnancy-related health, safe delivery, and postnatal care for themselves and their newborn. Gender inequality may lead to neonatal mortality through decreased access to education, economic opportunities and access to health care resources (WHO, 2004). Women and girls may also experience exposures and vulnerabilities that are poorly recognized in society (WHO, 2004), e.g., husband decides on contraception, resulting in low contraceptive use and close birth spacing; and husband's justification of wife-beatings that may lead to abuse resulting in maternal morbidity, premature labour and/or complications of labour and neonatal mortality. How husband's justification of abuse translates to acts of abuse' is an important area of research that is understudied, in part because most countries do not collect these types of data. In summary, when mothers and girls are treated as inferior by others and denied their basic human rights, pregnancy health and safe delivery are at risk, increasing the likelihood of neonatal mortality.

The purpose of this study is to identify where to target SDGinterventions to reduce neonatal mortality in two highly prevalent sub-regions of Africa, i.e. East Africa and West Africa. Interventions focus on three dimensions of need: i) where to improve care around the time of delivery; ii) where to increase mother's education; iii) where to further empower women.

\section{Materials and Methods}

A cross-sectional retrospective study design was used to study the Demographic and Health Survey (DHS) birth and infant death data (http://dhsprogram.com/data/Data-Variables-and-Definitions. $\mathrm{cfm}$ ) for countries in East Africa and West Africa. Geographically weighted Poisson regression (GWPR) models are estimated to spatially match these needs with high-neonatal mortality districts within countries in East Africa and West Africa.

\section{Study areas}

Seven countries in East Africa with a total of 69 districts were included in the study, including Ethiopia $(n=11)$, Kenya $(n=8)$, Malawi $(n=3)$, Mozambique $(n=10)$, Tanzania $(n=19)$, Zambia $(\mathrm{N}=10)$ and Zimbabwe $(\mathrm{n}=8)$. In West Africa, there were also 7 countries with a total of 62 districts in the study, including Benin $(n=12)$, Burkina Faso $(n=13)$, Ghana $(n=10)$, Guinea $(n=8)$, Mali $(n=6)$, Niger $(n=7)$ and Nigeria $(n=6)$.

\section{Data}

The DHS data for each country was requested and approved using the registration process at (http://dhsprogram.com/data/newuser-registration.cfm). The most recent years of DHS survey data were used in this study, including East Africa: Ethiopia (2011), Kenya (2008-2009), Malawi (2010), Mozambique (2011), Tanzania (2010), Zambia (2013-2014) and Zimbabwe (20102011); and West Africa: Benin (2011-2012), Burkina Faso (2010), Ghana (2014), Guinea (2012-2013), Mali (2012-2013), Niger (2012) and Nigeria (2013). While the more widespread regional designation for Malawi, Mozambique and Zimbabwe is Southern
Africa, this study adopted the UN designates for these countries in East Africa. The DHS Birth Recode datasets were used, comprising all children ever born to interviewed women in the study areas $(n=630,125)$. Neonatal deaths were defined as infant deaths that occurred 0 to 30 days to all live singleton births in the first year of life (0 to 12 months). The births and neonatal deaths were calculated using a synthetic cohort life table approach (Rutstein and Rojas Guide to DHS Statistics, 2006) from which neonatal mortality rates were calculated. To construct this synthetic cohort - the dates of mother's interviews were identified and all births occurring 10 years prior to those interview dates were calculated. In addition, 9 months of births were added at the beginning of the 10-year time period ( 9 months prior to mother's interviews) to capture those births of neonatal deaths that occurred within the 10 year time period. Furthermore, 9 months of births were added at the end of the 10 -year time period to capture those neonatal deaths of births that occurred within the 10 year time period. Nine months were used on each end of the 10 year time period to capture a full gestational period. Within this synthetic cohort $-100 \%$ of births and neonatal deaths were counted within the 10 year time period and one-half of births and neonatal deaths were counted within the two 9 month extended time periods to account for infant's partial exposure to mortality (Rutstein and Rojas, 2006). After removing births not included in this synthetic cohort of infants, the dataset was reduced to 361,738 births. Only live singleton births were studied, further reducing the dataset to 348,482 births. Finally, only resident mothers were studied, leaving the final dataset with 344,264 births, including East Africa, $\mathrm{n}=149,683(44.2 \%)$ and West Africa, $\mathrm{n}=194,581(55.7 \%)$.

\section{Final datasets}

The neonatal mortality rates were calculated using the unweighted sum of the synthetic cohort of neonatal deaths divided by the sum of live births for each district, country and region (Cohort dataset - unweighted). In the descriptive analyses - the weighted frequencies of maternal and infant characteristics were calculated using the complete cross-section of births during the full 10 years and two 9-month extended time periods (Cross-sectional dataset - weighted). The weights (assigned per cluster, stratum and individual) were provided in the DHS dataset. In subsequent GWPR analyses - the unweighted frequencies of maternal and infant characteristics as described below were analysed using the same cross-section of data (Cross-sectional dataset - unweighted). Please see Table 1 for a summary of these study datasets. For a complete description of the DHS sampling methodology and weighting scheme please refer to Rutstein and Rojas (2006).

The dependent variable in the GWPR models was the count of neonatal deaths (Poisson model) in each district offset by the expected number of neonatal deaths. The expected number of neonatal deaths was calculated using indirect standardisation - i.e., the number of births in each district multiplied by the neonatal mortality rate for developing countries (22 deaths per 1000 live births) (WHO, 2015a). The independent variables were three risk factors for neonatal mortality each describing the dimensions of need (1-3 a,b,c) including: (1a) the percentage of mothers' who delivered at home (MDelHome), (1b) the percentage of infants' delivered without a doctor or health professional (MDelNotDoc), and (1c) the percentage of mothers who received prenatal care with an attendant who was not a doctor or health professional (MPreNotDoc); and (2a) the percentage of mothers whose highest education was no education (MEducNo), (2b) the percentage of mothers whose highest educa- 
tion was a primary education - excluding those mothers with no education (MEducPr), and (2c) the percentage of mothers who were illiterate (MIllit) (Note: the difference between 2a and 2c is that mother's may be literate (read and write) without a primary education if they are taught at home or by someone in a facility outside of the education sector); and (3a) the percentage of mother's who did not want or plan to have their last child (MNotWtChld), (3b) the percentage of mother's whose husband decided on their contraceptive practices (HDecCont), and (3c) the percentage of mother's whose husband justified wife-beating (HBeat). Other maternal and infant characteristics that were used as control variables in the GWPR models included the percentage of young mothers 15 to 24 years excluding mothers 35-49 years (MAgeYng), the percentage of older mothers 35 to 49 years - excluding 15 to 24 years (MAgeOld), the percentage of mothers with a wealth index status of very poor or poor (MPoor), the percentage of mothers residing in rural areas (MRural), the percentage of mothers exposed to unprotected drinking water (MWater), the percentage of mothers who had more than one son and/or daughter die previous to this child (MSDied and MDDied), the percentage of female infants (CFemale), the percentage of infants born of small size (CSmall) and the percentage of infants who were sick with fever, cough or diarrhea (CSick) in the two weeks prior to the mother's interview. The potential for multicollinearity among these variables and how it was addressed in this study are discussed in the Methods Section. The geographic data used in this study, consisted of shapefiles by ArcGIS v.10.2 [Environmental Systems Research Institute (ESRI), 2015] for all districts in countries in East Africa and West Africa. These files were requested and obtained from the Minnesota Population Center and ICF International, Integrated Demographic and Health Surveys (https://www.idhsdata.org/idhs). The frequencies of neonatal deaths and births were calculated for each district to ensure an adequate sample ( $>50$ births and 20 neonatal deaths) (Hamilton et al., 2006) for subsequent analyses. Two districts in Mozambique (Inhambane and Gasa) were merged to capture 24 neonatal deaths, three districts in Tanzania (Arusha, Kilmanjaro, Manyara) were merged to capture 25 neonatal deaths and three districts in Zimbabwe (Bulawayo, Matabeleland North and South) were merged to capture 24 neonatal deaths. The study used a Robinson world projection and World Geodetic coordinate system 1984 in meters to ensure optimal distance measures across East Africa and West Africa study regions in subsequent GWPR analyses.

Table 1. Overview of study datasets by region, demographic and health survey data-birth recode data.

\begin{tabular}{lccc} 
& $\begin{array}{c}\text { East } \\
\text { Africa (n) }\end{array}$ & $\begin{array}{c}\text { West } \\
\text { Africa (n) }\end{array}$ & $\begin{array}{c}\text { Total } \\
\text { (n) }\end{array}$ \\
Original dataset & 278,622 & 351,503 & 630,125 \\
After defining study time period & 158,121 & 203,617 & 361,738 \\
\hline After removing multiple births & 152,588 & 195,894 & 348,482 \\
After removing visiting mothers $^{*}$ & 149,683 & 194,581 & 344,264 \\
Cohort dataset (unweighted) $^{*}$ & 143,567 & 187,276 & 330,843 \\
$\quad$ Neonatal deaths & 4835 & 6334 & 11,169 \\
Cross-sectional dataset (weighted) & & & \\
$\quad$ & 150,511 & 196,616 & 347,127 \\
$\quad$ Neonatal deaths & 5346 & 6654 & 11,900 \\
\hline${\text { Cross-sectional dataset (unweighted) }{ }^{\#}}^{\text {Neonatal deaths }}$ & 146,658 & 194,581 & 341,239 \\
\hline
\end{tabular}

*Dataset used to calculate neonatal mortality rates; ${ }^{\circ}$ dataset used to calculate frequencies of maternal and infant characteristics; "dataset used to conduct geographically weighted poisson regression analyses.

\section{Methods}

Preliminary data analyses for this study included checking the univariate and bivariate spatial autocorrelation in the dependent and independent variables using the local Moran's I (Anselin, 1991) to minimise bias in subsequent GWPR models (Wheeler and Tiefelsdorf, 2005). The global Moran's I was also used to assess the overall level of clustering in the final models. These analyses were conducted using GeoDa freeware, software obtained at: https://geodacenter.asu.edu/

GWPR models were estimated to capture the spatially varying relationships between the dimensions of need and high-neonatal mortality districts in order to target specific health care and public health interventions in those districts of greatest need. GWPR models differ from generalised linear models because the parameters $\beta_{0}, \ldots, \beta_{k}$ estimated at regression points are allowed to vary by locations (in this study locations were defined as the spatial coordinates at the centroid of each district). The calibration of GWPR models therefore, requires selecting a kernel type and bandwidth to be applied at each regression point from within which to capture the maternal and infant data at each location. Kernel types are based on a distance-based weighting scheme (Gaussian or bisquare) whereby the measurements of locations close to the regression point are given more weight than measurements farther away - allowing for the smoothing of regression parameters (Fotheringham et al., 2002) (Figure 1). Gaussian kernels weight measurements continuously and gradually decrease from the kernel centre but never reaches zero. The bi-square kernel has a defined range by which to determine local extents for model fitting. The kernel's bandwidth can be set to fixed (a fixed-sized) or adaptive (a size-varying bandwidth). Gaussian kernel types are often fixed to avoid having no data measurements within the kernel. Adaptive bandwidths may be used if there is substantial spatial variation in the distribution of population at risk. The GWPR model was constructed as:

$y_{i} \sim$ Poisson $\left.\left[N_{i} \exp \left(\sum_{k} \beta_{k} u_{i}, v_{i}\right) x_{k, j}\right)\right]$

Eq. 1

where the dependent variable $y_{i}$ neonatal deaths was a count variable assumed to be Poisson distributed. $N_{i}$ was the offset variable to define the size of the population at risk at $i$ th location. $\beta_{k}$ was the independent variable-slope coefficients varying conditional on the

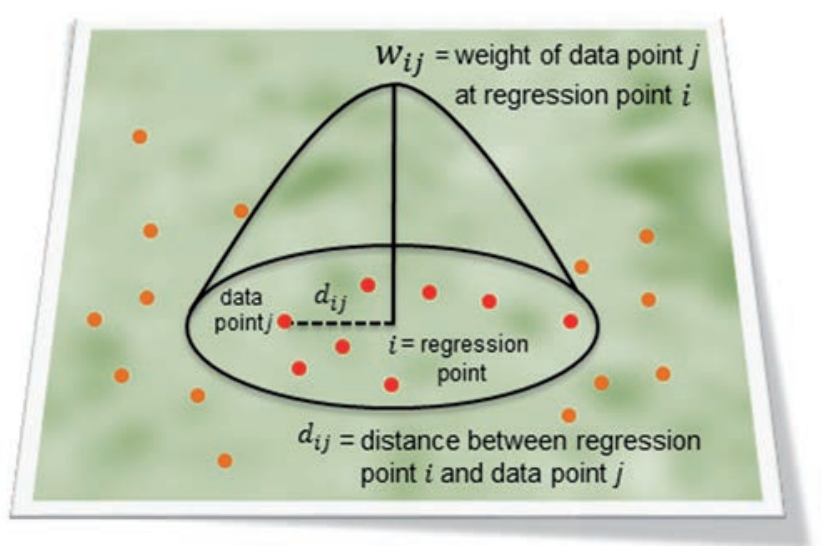

Figure 1. Fixed spatial kernel. 
location $\left(u_{i}, n_{i}\right)$ the $\mathrm{x}, \mathrm{y}$ coordinates of the th location. In non-parametric models, the first variable was constant by setting $x_{0 j}=1$, after which $\beta_{0}\left(u_{i}, n_{i}\right)$ became a geographically varying intercept term.

Semiparametric GWPR models (SGWPR) were estimated to study the independent contribution of variables that represent each dimension of need on neonatal mortality, controlling for potential differences in maternal and infant characteristics and known health risk factors.

$$
\begin{aligned}
& y_{i} \sim \text { Poisson }\left[N_{i} \exp \left(\sum_{k} \beta_{k} u_{i,}, v_{i}\right) x_{k, j}+\right. \\
& \left.\left.\sum_{i} y_{l} z_{l, i}\right)\right]
\end{aligned}
$$

where $z_{l, i}$ was the $l$ th independent variable with a fixed (global) coefficient $y_{l}$. This model mixed geographically local and global terms. The intercept term was specified as a varying term in case other coefficients varied, causing variation in the intercept. The SGWPR modelling parameters included fixed and bi-square kernels for East Africa and West Africa.

Fitting GWPR models also involves a geographical variability test that uses the kernel bandwidths to compare $k$ th varying coefficient(s) parameters over space. In this study, the model comparison criterion was the default Akaike Information Criterion (AICc) used to determine the best-fit model. First, the AICc from a global model and GWPR models were compared and if the GWPR model AICc decreased (greater than 2) the model was considered to perform very differently - indicative of spatial variation in the dependent and independent variables and the need for GWPR.

The output intercepts and slope coefficients were standardised and converted to odds ratios (OR) to report the odds of neonatal mortality for mother's residing in districts with varying needs. The intercept and slope coefficients and the residuals from the best-fit models were mapped in ArcGIS version 10.2 using a choropleth map design. The classification scheme for the intercept coeffi- cients was constructed similarly to compare the estimated district neonatal mortality rates across East and West Africa. The slope coefficient distributions did not lend themselves to similar classification schemes to compare model results; instead a natural break classification scheme was applied to each distribution. Finally, the pseudo-t test accompanying each estimated independent variable was mapped to show the levels of significance of the slope coefficients and where the model over-estimated (pseudo-t $<-1.96$ ) or under-estimated (pseudo-t $>1.96$ ) neonatal mortality. Those districts with elevated neonatal mortality rates and slope coefficients $(\mathrm{OR}>1.0$ and pseudo-t $>1.96)$ were considered districts in significant need of future SDG-intervention(s).

The SGWPR analyses were conducted in GWR4.0 freeware, software developed at the National Centre for Geocomputation, National University of Ireland Maynooth (http://gwr.nuim.ie) and Department of Geography, Ritsumeikan University, Japan (http://www.ritsumei.ac.jp/acd/cg/lt/asp/eng/studying/index.html). The software was downloaded from the GeoDa Center for Geospatial Analysis and Computation website (https://geodacenter.asu.edu/gwr_software). For another example of an application of GWR in a health study, please refer to Feuillet et al. (2015). The descriptive results and the findings from the SGWPR models are outline below.

\section{Results}

Table 2 shows the overall numbers of births, neonatal deaths and neonatal mortality rates for the countries and two regions. West Africa had the highest number of neonatal deaths $(n=6334)$ but the neonatal mortality rates were relatively similar for West Africa $(33.8,95 \%$ CI 32.9-34.6) and East Africa (33.6, 95\% CI 32.7-34.6). The standardised neonatal mortality ratio (SMR) was

\begin{tabular}{|c|c|c|c|c|c|c|}
\hline Region and country & Districts (n) & $\begin{array}{c}\text { Infants } \\
\leq 1 \text { year }(n)\end{array}$ & $\begin{array}{l}\text { Neonatal } \\
\text { deaths* (n) }\end{array}$ & $\begin{array}{l}\text { Neonatal } \\
\text { mortality }{ }^{\circ} \# \\
\text { n (rate) }\end{array}$ & $\begin{array}{c}\text { Expected } \\
\text { neonatal } \\
\text { deaths }{ }^{\S}(\mathrm{n})\end{array}$ & $\begin{array}{c}\text { Standardised } \\
\text { mortality } \\
\text { ratio }^{\wedge}\end{array}$ \\
\hline East Africa & 69 & 143,567 & 4835 & $33.6(32.7-34.6)$ & 3160 & 1.53 \\
\hline Ethiopia & 11 & 23,457 & 1003 & $42.7(40.0-45.4)$ & 516 & 1.94 \\
\hline Kenya & 8 & 11,477 & 362 & $31.5(28.2-34.8)$ & 252 & 1.43 \\
\hline Malawi & 3 & 38,371 & 1244 & $32.4(30.5-34.2)$ & 1074 & 1.47 \\
\hline Mozambique & 10 & 20,194 & 681 & $33.7(31.1-36.3)$ & 444 & 1.53 \\
\hline Tanzania & 19 & 14,545 & 724 & $49.7(46.0-53.4)$ & 409 & 1.77 \\
\hline Zambia & 10 & 25,752 & 590 & $22.9(21.0-24.7)$ & 566 & 1.04 \\
\hline Zimbabwe & 8 & 9770 & 230 & $23.5(20.4-26.6)$ & 214 & 1.07 \\
\hline West Africa & 62 & 187,276 & 6334 & $33.8(32.9-34.6)$ & 4362 & 1.45 \\
\hline Benin & 12 & 25,921 & 567 & $21.8(20.0-23.7)$ & 570 & 0.99 \\
\hline Burkina Faso & 13 & 29,715 & 982 & $33.0(30.9-35.1)$ & 653 & 1.50 \\
\hline Ghana & 10 & 11,142 & 324 & $29.0(25.8-32.2)$ & 245 & 1.32 \\
\hline Guinea & 8 & 13,921 & 521 & $37.4(34.1-40.6)$ & 306 & 1.70 \\
\hline Mali & 6 & 19,846 & 755 & $38.0(35.3-40.8)$ & 436 & 1.72 \\
\hline Niger & 7 & 25,391 & 732 & $28.8(26.7-30.9)$ & 558 & 1.35 \\
\hline Nigeria & 6 & 61,338 & 2452 & $39.9(38.3-41.5)$ & 1349 & 1.81 \\
\hline
\end{tabular}

Table 2. Neonatal mortality rates and standardised mortality ratios by region and country: demographic and health survey data.

year*Developing Regions rate, 22 per 1000 in 2013) (UNICEF, 2015); ^ standardised mortality ratios (observed neonatal deaths/expected neonatal deaths). Birth recode data from most recent survey year by country. 
higher in East Africa (1.53) compared to West Africa (1.45); however, both regions had higher neonatal mortality than developing countries as a whole. The highest neonatal mortality rates and SMRs in countries across the regions were Tanzania (49.7, 95\% CI 46.0-53.4, SMR=1.77), Ethiopia (42.7, 95\% CI 40.0-45.4, $\mathrm{SMR}=1.94)$ and Nigeria $(39.9,95 \%$ CI $38.3-41.5, \mathrm{SMR}=1.81)$. In contrast, the lowest neonatal mortality rates and SMRs were in Benin $(21.8,95 \%$ CI 20.0-23.7, SMR=0.99), Zambia $(22.9,95 \%$ CI 21.0-24.7, SMR=1.04) and Zimbabwe (23.5, 95\% CI 20.4-26.6, $\mathrm{SMR}=1.07)$. Thus, four countries with the highest and lowest neonatal mortality rates were in East Africa - demonstrating the substantial variability in neonatal mortality rates across this region.

Table 3 shows the frequencies for two of the most significant dimensions of need to reduce neonatal mortality by country and region. In terms of care around the time of delivery the region with the highest percentage of home births was West Africa (48.6\%) followed by East Africa (38.4\%). In both regions a substantial proportion of births delivered were without a doctor, midwife or health professional (West Africa, 44.4\%) and (East Africa, 32.9\%). Ethiopia had over $50 \%$ of home births and births delivered without a doctor, midwife or health professional. Those countries with a high home delivery rate but differing percentages of births delivered without a doctor, midwife or health professional included Niger (70.3 and 38.7\%), Nigeria (64.4 and 37.1\%), Guinea (59.3 and $25.7 \%$ ), Kenya (57.0 and 28.1\%) and Tanzania (49.1 and $51.7 \%$ ). These findings demonstrate the need to further understand the drivers behind home births and the need to further invest in the medical training of doctors, midwives and other health care providers in these regions.

In terms of mother's education, the region with the highest percentage of mothers with no education was West Africa (70.3\%) followed by East Africa (29.5\%). All countries in West Africa except Ghana had over half of mothers with no education. In East Africa, Ethiopia and Mozambique had the highest percentages of mothers with no education $(72.9 \%, 40.3 \%)$. Instead, East Africa had a higher percentage of mothers with primary education (53.6\%) compared to West Africa (14.2\%). These findings show the need for education and health awareness particularly for women of reproductive ages in all countries of West Africa and Ethiopia and Mozambique in particular, in East Africa.

Finally, in terms of the empowerment of women the region with the highest percentage of mothers who did not want or plan to have their last child was East Africa (37.8\%) followed by West Africa (13.2\%). Approximately half of mothers in Malawi (51.5\%) and Kenya $(50.0 \%)$ did not want or plan to have their last child. Both regions had a high percentage of husbands who justified wife-beating (West Africa, 48.6\%) and (East Africa, 42.0\%) (data not shown in Table 3). In East Africa there were four countries in which over $50.0 \%$ of husbands justified wife-beating including, Ethiopia (77.4\%), Kenya (58.8\%), Tanzania (58.6\%) and Zambia $(51.8 \%)$ and in West Africa those percentages were even higher, Guinea (94.3\%), Mali (78.6\%) and Niger (61.8\%). The reasons given to justify wife-beatings included: Goes out without telling husband, perceived neglect of children, wife argues with husband, wife refuses to have sex with husband, and/or if wife burns the food. These findings show the widespread need for interventions to further empower women and reduce domestic violence across both regions.

The GWPR and SGWPR analysis results are shown in Table 4. There was a sharp reduction in the AICc from the global to the GWPR unadjusted models in both regions, demonstrating the need to model the spatial variation in neonatal mortality at the district level. First, the spatial variation in neonatal mortality rates, controlling for risk factors representing the three dimensions of need

Table 3. Descriptive frequencies of sustainable development goals-interventions to reduce neonatal mortality by region and country: demographic and health survey data.

\begin{tabular}{lcccccc} 
Region and country & $\begin{array}{c}\text { Care around delivery } \\
\text { Delivery } \\
\text { birth at } \\
\text { home }\end{array}$ & $\begin{array}{c}\text { Attendant } \\
\text { not doctor, midwife } \\
\text { or health professional }\end{array}$ & $\begin{array}{c}\text { Mother's education } \\
\text { no education }\end{array}$ & $\begin{array}{c}\text { Mother } \\
\text { primary } \\
\text { education }\end{array}$ & $\begin{array}{c}\text { Wother did not } \\
\text { want child }\end{array}$ & $\begin{array}{c}\text { Husband decided } \\
\text { contraception }\end{array}$ \\
East Africa & $38.4(71,655)$ & $32.9(71,805)$ & $29.5(150,487)$ & $53.6(150,487)$ & $37.8(127,417)$ & $11.4(51,037)$ \\
Ethiopia & $89.6(11,266)$ & $81.6(11,263)$ & $72.9(24,974)$ & $23.9(24,974)$ & $33.5(21,479)$ & $4.0(5860)$ \\
\hline Kenya & $57.0(5604)$ & $28.1(5605)$ & $13.8(11,778)$ & $63.4(11,778)$ & $50.0(9859)$ & $13.8(4251)$ \\
Malawi & $24.3(18,499)$ & $12.4(18,514)$ & $20.3(39,331)$ & $67.0(39,331)$ & $51.5(34,455)$ & $9.4(16,713)$ \\
\hline Mozambique & $2.7(10,978)$ & $29.3(11,133)$ & $40.3(22,263)$ & $50.1(22,263)$ & $15.0(19,692)$ & $17.8(2288)$ \\
Tanzania & $49.1(7413)$ & $51.7(7412)$ & $26.0(15,079)$ & $69.4(15,079)$ & $30.1(13,186)$ & $10.0(4515)$ \\
\hline Zambia & $31.5(12,615)$ & $18.8(12,596)$ & $12.6(26,786)$ & $58.5(26,786)$ & $40.5(23,418)$ & $16.3(11,599)$ \\
Zimbabwe & $34.0(5280)$ & $20.7(5280)$ & $2.4(10,275)$ & $35.1(10,275)$ & $35.8(8405)$ & $11.7(5742)$ \\
\hline West Africa & $48.6(92,014)$ & $44.4(91,844)$ & $70.3(19,6571)$ & $14.2(196,571)$ & $13.2(173,712)$ & $48.7(195,520)$ \\
Benin & $13.1(12,446)$ & $11.3(12,446)$ & $75.2(26,163)$ & $15.4(26,163)$ & $19.9(22,540)$ & $17.4(25,980)$ \\
\hline Burkina Faso & $33.4(14,611)$ & $23.0(14,598)$ & $86.4(31,563)$ & $9.4(31,563)$ & $9.4(28,188)$ & $47.1(31,551)$ \\
Ghana & $27.4(5270)$ & $10.4(5270)$ & $30.5(11,092)$ & $20.3(11,092)$ & $33.5(9102)$ & $32.0(11,076)$ \\
\hline Guinea & $59.3(6614)$ & $25.7(6588)$ & $81.9(14,419)$ & $10.3(14,419)$ & $18.1(12,293)$ & $94.32(14,412)$ \\
Mali & $43.6(10,044)$ & $14.7(10,044)$ & $85.3(20,850)$ & $8.0(20,850)$ & $14.9(18,752)$ & $78.6(20,731)$ \\
\hline Niger & $70.3(12,810)$ & $38.7(12,793)$ & $87.7(27,853)$ & $8.9(27,853)$ & $9.6(25,643)$ & $61.8(27,691)$ \\
Nigeria & $64.4(30,219)$ & $37.1(30,104)$ & $52.4(64,630)$ & $20.2(64,630)$ & $9.0(56,653)$ & $39.3(64,079)$ \\
\hline
\end{tabular}

Weighted frequencies (cross-section 10 years post-interview survey); birth recode data from most recent survey year by country. Values are expressed as percentage (n). 
are presented. Thereafter, the district locations with significant dimensions of need for countries and regions are described below.

\section{Neonatal mortality}

In East Africa, the observed neonatal mortality rates by district ranged from 11.0 to 102.0 per 1000 live births (Figure 2A). Nine of the 15 districts with the highest neonatal mortality rates $(>50.0)$ were in Tanzania, including Lindi (102.4) and neighboring coastal districts (Dar es Salaam, Mtwara, Pwani and Tanga), contiguous interior districts (Morogoro and Iringa) and districts in southwest (Rukwa) and northwest (Mara) Tanzania. In addition, there were exceptionally high rates of neonatal mortality in Ethiopia, districts northwest of Addis Ababa (Amhara, Benishangui-Gumuz) and in Mozambique (Tete and Zambezia). Districts with neonatal mortality rates above 30.0 were found throughout the region. The estimated neonatal mortality rates in East Africa (Figure 2B) ranged from 15.4 to 61.0 , controlling for complications arising from home deliveries, mothers with no education, mothers whose husbands decided on contraceptive practices and rural residency (Table 4). In Tanzania's coastal districts, the estimated neonatal mortality rates declined from the observed substantially in Lindi (from 102.4 to 48.4) and Mara (80.0 to 46.0) and in Mozambique's districts, Tete (50.5 to 23.0) and Zambezia (53.4 to 20.1), respectively. The capital city of Mozambique also experienced a substantial decline in observed-to-estimated neonatal mortality (Maputo, 41.6 to 16.7).

In West Africa (Figure 3A) the observed neonatal mortality rates by district ranged from 8.4 to 48.1 deaths per 1000 live births. The district with the highest neonatal mortality rate was Est in Burkina Faso (48.1). Other districts with observed neonatal mortality rates above 40.0 included Cascades and Sahel also in Burkina Faso, Ashanti in Ghana, Sikasso in Mali, Mamou, Labe and Kankan in Guinea and Nigeria's North East, North West and South East districts. The estimated neonatal mortality rates in West Africa ranged from 8.4 to 44.9 , controlling for the spatial variation in complications arising from home deliveries, mothers with a primary education and mothers who reported not wanting to have their last child (Table 4). The estimated neonatal mortality rates
(Figure 3B) declined steadily from the observed in Est, Burkina Faso (48.1 to 33.5 ), while other countries showed declines that were less dramatic, including Cascades (43.8 to 33.2) and Sahel (41.7 to 38.3) in Burkina Faso, Ashanti in Ghana (47.7 to 26.9), Sikasso in Mali (47.3 to 41.5) and there were smaller declines in Mamou (46.8 to 44.5) and Labe (43.0 to 39.2) in Guinea. The estimated neonatal mortality rate also declined from those observed in Nigeria's South East district (40.0 to 32.0) but not in the North West (44.8) or North East (44.7) districts, respectfully.

The global Moran's I did not show substantial spatial autocorrelation in neonatal mortality rates across districts in East Africa or West Africa indicating that this was not a factor in the estimation of the SGWRP models. The results were therefore, relatively robust at the district level across countries in both regions.

\section{Care around time of delivery}

In East Africa (Figure 4A), infants delivered at home were at highest odds of neonatal mortality in the districts of Cabo Delgado and Nampula $(\mathrm{OR}=1.4)$ in northeastern Mozambique, controlling for complications arising in mothers with no education, mother's whose husbands decided on their contraceptive practices and rural residency. Generally, infants born at home were at increased odds of mortality across the East Africa region with significantly high districts in Ethiopia, particularly areas in the Somalia Regional State in eastern Ethiopia, districts in central Tanzania, northern Zambia, northern and southern Malawi and northern and central Mozambique. Importantly, the pseudo-t was highest $(t>6.0)$ in three districts in Tanzania, specifically Lindi and Mtwara on the coast and Morogoro in the interior. Of importance, neonatal complications associated with home delivery that led to deaths in East Africa were largely explained by maternal exposure to unprotected drinking water sources. In West Africa (Figure 4B) infants delivered at home were at highest odds of neonatal mortality in Niamey, the capital of Niger $(\mathrm{OR}=2.6)$, controlling for complications arising in mothers with a primary education and mothers who did not want to have their last child. The odds ratio and pseudo-t were highest ( $\mathrm{t}=7.94$ to 10.3 ) in districts in northern Benin (Alibori,

Table 4. Semiparametric geographically weighted Poisson regression analyses estimating neonatal mortality at the district level within regions: demographic and health survey data.

\begin{tabular}{|c|c|c|c|c|c|}
\hline Model & Bandwidth & Deviance & K & $\mathrm{AICc}$ & Diff AlCc \\
\hline \multicolumn{6}{|l|}{ East Africa } \\
\hline Global PR & na & 599.6 & 1.0 & 601.6 & 230.4 \\
\hline GWPR & 914048 & 354.2 & 7.5 & 371.2 & 16.9 \\
\hline SGWPR (MDelHome) & 981380 & 330.2 & 10.1 & 354.2 & 26.5 \\
\hline SGWPR (MDelHome; MEducNo) & 1015257 & 292.6 & 13.7 & 327.6 & 18.2 \\
\hline SGWPR (MDelHome; MEducNo; HDecCont) & 1093768 & 263.9 & 16.8 & 309.4 & 27.5 \\
\hline SGWPR (MDelHome; MEducNo; HDecCont; MRural)* & 1126860 & 255.8 & 19.6 & 281.9 & 16.0 \\
\hline SGWPR (MDelHomeNS; MEducNo; HDecCont; MRural; MWater) & 1183566 & 202.9 & 21.3 & 265.9 & 0.0 \\
\hline \multicolumn{6}{|l|}{ West Africa } \\
\hline Global PR & na & 459.7 & 1.0 & 461.8 & 196.0 \\
\hline GWPR & 581264 & 246.2 & 8.2 & 265.7 & 25.2 \\
\hline SGWPR (MDelHome) & 648579 & 211.1 & 11.6 & 240.4 & 25.6 \\
\hline SGWPR (MDelHome; MEducPr) & 671750 & 175.5 & 14.6 & 214.7 & 2.7 \\
\hline SGWPR (MDelHome; MEducPr; MNotWtChld) ${ }^{\circ}$ & 785184 & 169.3 & 15.5 & 212.0 & 21.0 \\
\hline SGWPR (MDelHome ${ }^{\text {NS; }}$ MEducPr; MNotWtChld; MAgeOld; CFemale) & 953098 & 139.4 & 17.7 & 190.9 & 0.0 \\
\hline
\end{tabular}

K, estimated parameters; AICc, small bias corrected Akaike Information Criterion; PR, Poisson regression; na, not available; GWPR, geographically weighted Poisson regression; SGWPR, semiparametric geographically weighted Poisson regression; MDelHome, mother delivered at home; MEducNo, mother's highest education was no education; HDecCont, husband decides on contraceptive practices; MRural, mother resided in a rural area; MWater, mother's water was not protected; MEducPr, mother's highest education was primary education; MNotWtChld, mother did not want child then or later; MAgeOld, mother was 35-49 years of age; CFemale, sex of infant was female. *Figures $1,4 \mathrm{~A}, 5 \mathrm{~A}, 6 \mathrm{~A} ;{ }^{\circ}$ Figures 2, 4B, 5B, 6B. 
Atacora, Borgou and Donga), Tahoua, Niger and North West, Nigeria. It is worth emphasising that neonatal complications associated with home births that led to deaths in West Africa were largely explained by mothers of older age and female infants.

\section{Education}

In East Africa (Figure 5A), the odds of neonatal mortality for

A

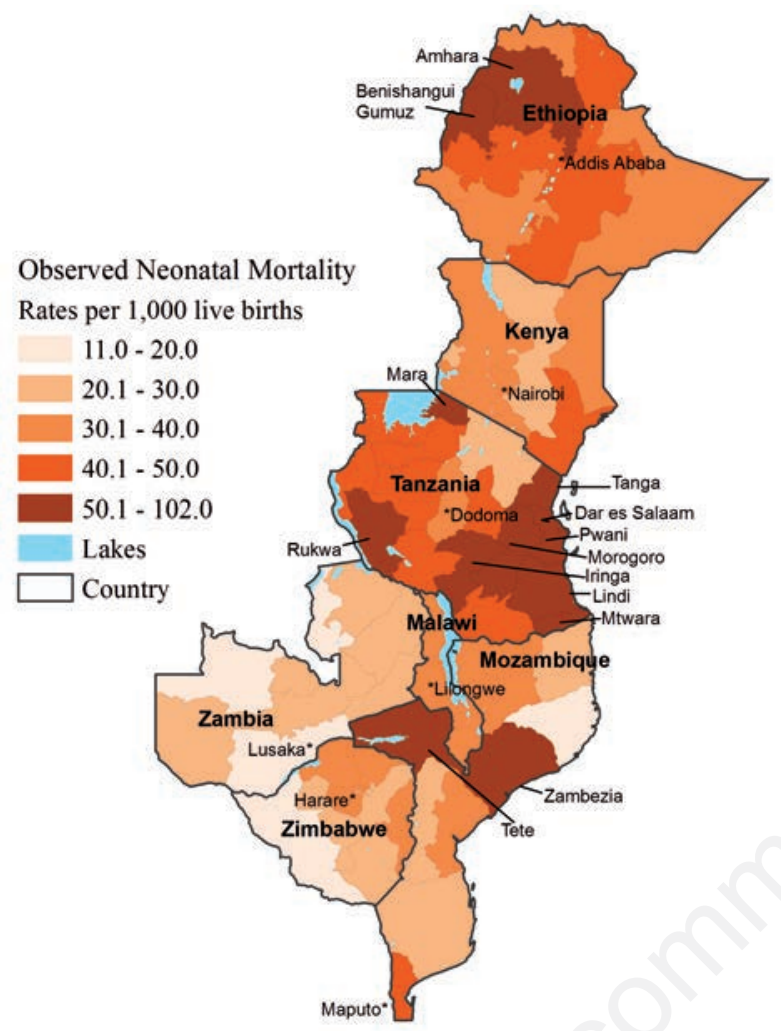

mothers with no education compared to all other mothers were greatest in districts in Zambia (North-Western and Western $\mathrm{OR}=1.7$ and Southern $\mathrm{OR}=1.6$ ), in Zimbabwe (Mashonaland East, Central and West, range $\mathrm{OR}=1.3,1.4$ ) (pseudo-t tests 6.91 to 8.20 ) and in Ethiopia (Benishangui-Gumuz $\mathrm{OR}=1.5$ ) (pseudo-t tests 2.59 to 6.90), controlling for home births and women whose husband decided on contraception. In general, infants of mothers with no

B

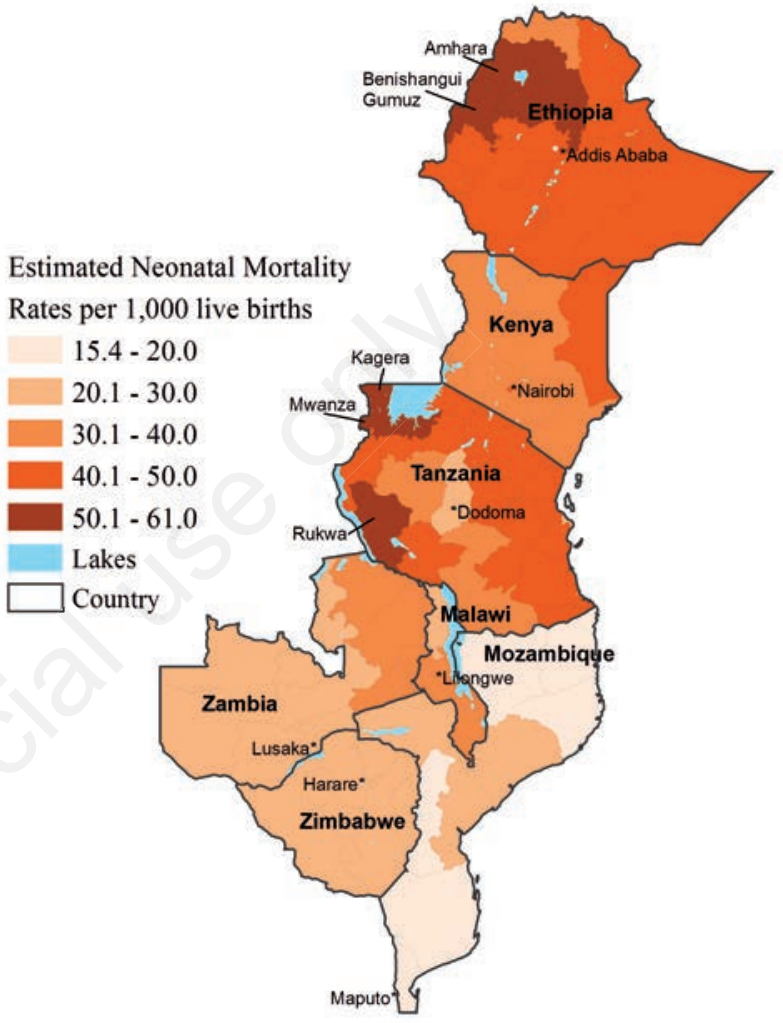

Figure 2. Observed (A) and estimated [controlling for MDelHome, MEducNo, HDecCont and MRural (66.2\% of deviance explained)] (B) neonatal mortality rates, geographically weighted Poisson regression, East Africa. Data were extracted from Birth Recode Data, the most recent survey year by country and region. Univariate Global Moran's I: estimated neonatal mortality rates=0.293.

A

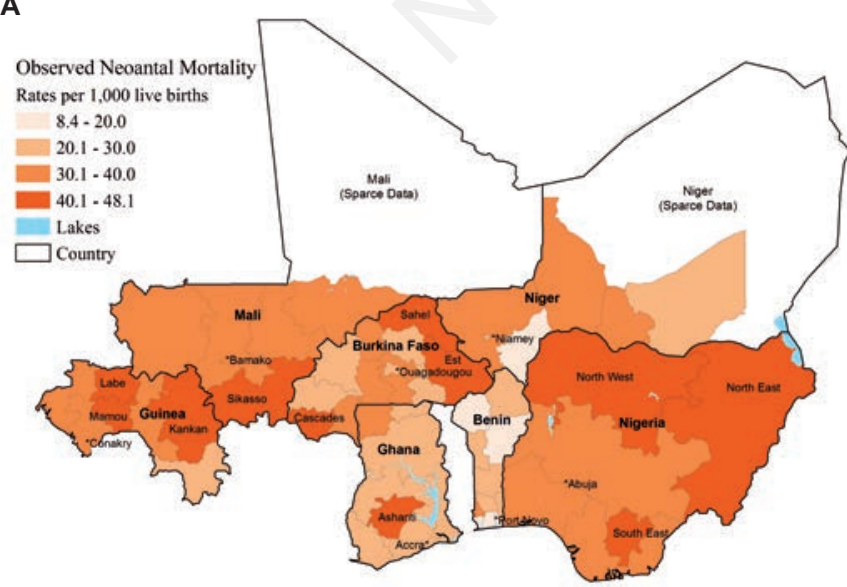

B

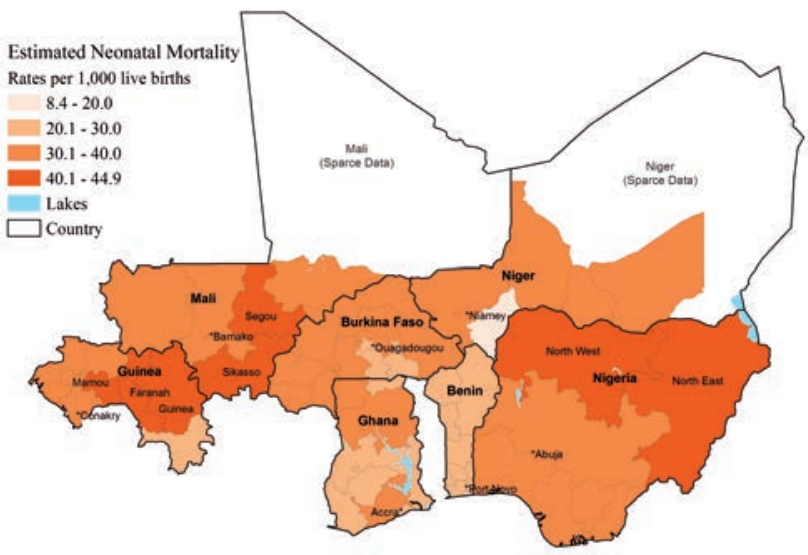

Figure 3. Observed (A) and estimated [controlling for MDelHome, MEducNo, HDecCont and MRural (70.0\% of deviance explained)] (B) neonatal mortality rates, geographically weighted Poisson regression, West Africa. Data were extracted from Birth Recode Data, the most recent survey year by country and region. Univariate Global Moran's I: estimated neonatal mortality rates=0.332. 
A

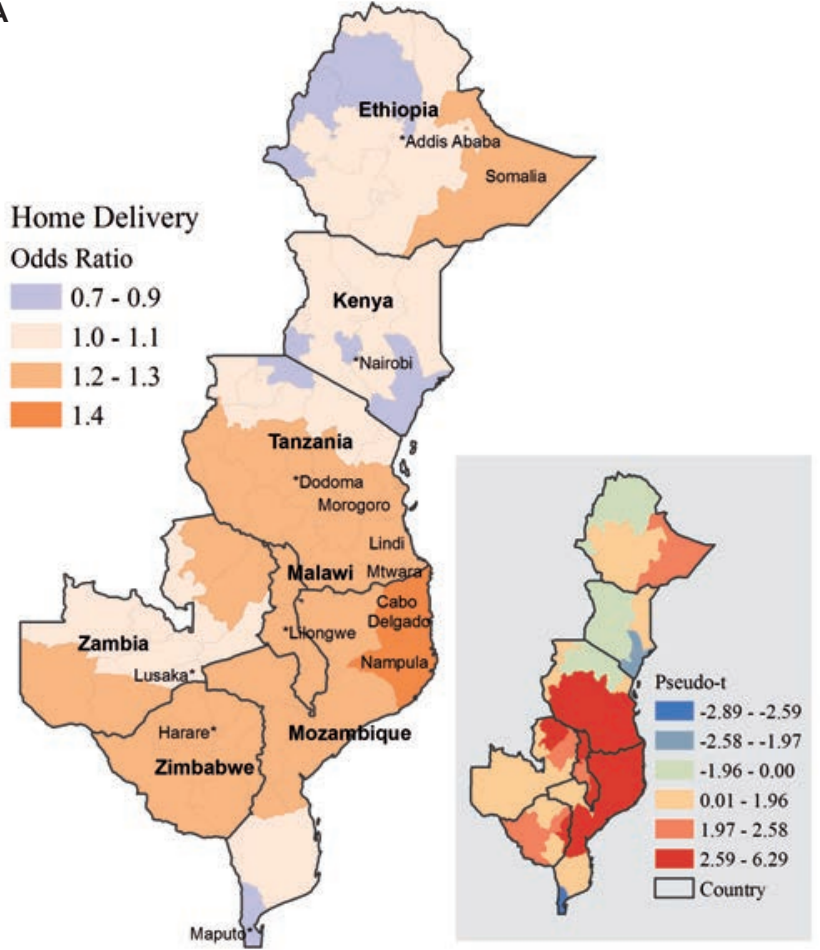

B

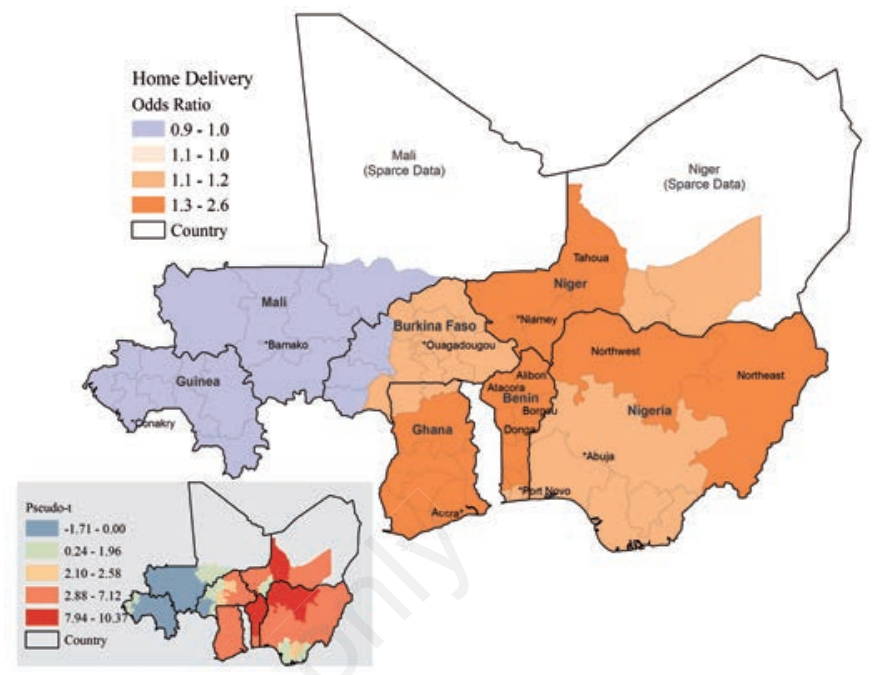

Figure 4. Estimated effects of care around the time of delivery dimension on neonatal mortality (controlling for variables, see Table 4), geographically weight Poisson regression analysis, East Africa (A) and West Africa (B). Data were extracted from Birth Recode Data, the most recent survey year by country and region. Bivariate Global Moran's I: East Africa=-0.060 and West Africa=0.144.

A

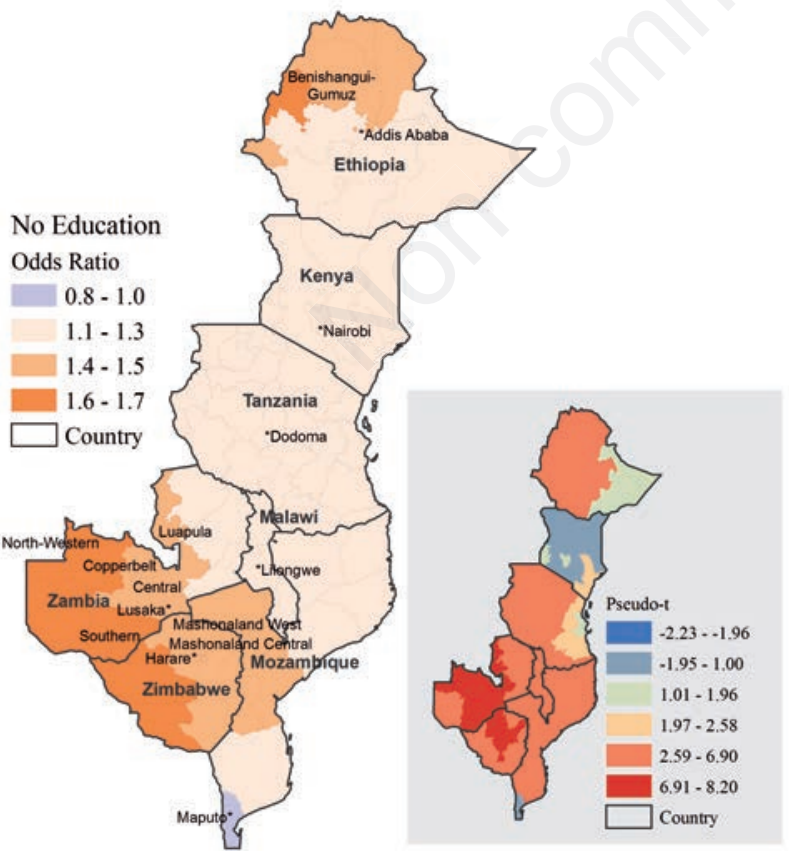

B

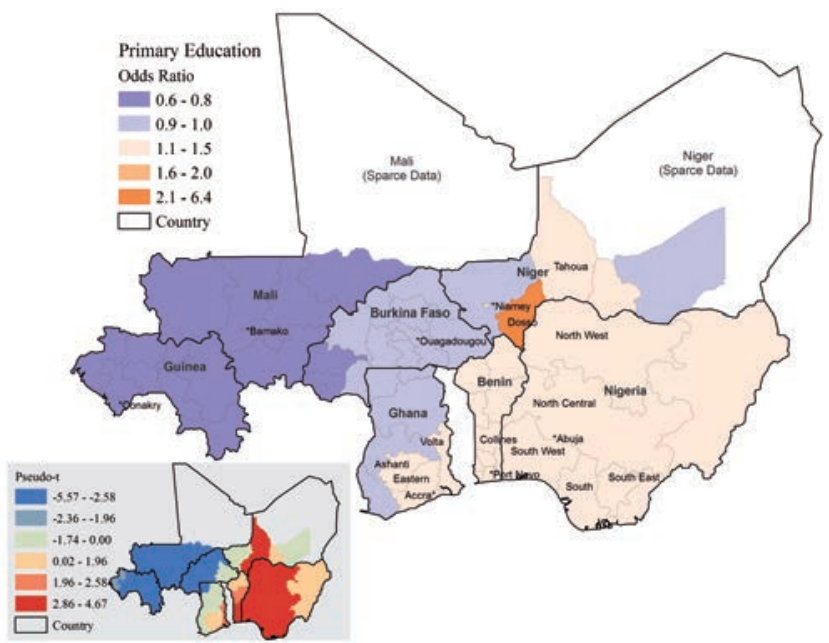

Figure 5. Estimated effect of mother's education dimension on neonatal mortality (controlling for variables, see Table 4), geographically weighted Poisson regression analysis, East Africa (A) and West Africa (B). Data were extracted from Birth Recode Data, the most recent survey year by country and region. Bivariate Global Moran's I: East Africa=0.035 and West Africa=0.111. 
education were at increased odds of neonatal mortality across East Africa, except in Kenya and Maputo, the capital city of Mozambique. Finally, being poor was not a significant risk factor for neonatal mortality - instead no education and rural residency appeared to explain neonatal mortality along the poverty-pathway. In West Africa (Figure 5B), infants of mothers with a primary education compared to mothers with secondary or higher education were at very high odds of neonatal mortality in the Dosso district of Niger $(\mathrm{OR}=6.4)$, Nigeria's districts (North West, North Central, South and South East) and southern districts in Benin (range, $\mathrm{OR}=1.1$ to 1.5 ). Districts in mid-Benin (north of Collines) and eastern Ghana, districts Volta, Eastern, Ashanti and Greater Accra, the capital of Ghana also had increased odds of neonatal mortality (range, $\mathrm{OR}=1.1$ to 1.5 ) (pseudo-t tests 2.56 to 4.87 ).

\section{Empowerment of women}

The types of empowerment needed for mothers varied across the regions. In East Africa (Figure 6A), infants of mothers whose husband's decided on their contraceptive practices were at increased odds of neonatal mortality in Ethiopia's districts Affar, Tigray, Amhara and Benishangui-Gumuz (range, $\mathrm{OR}=1.5$ to 1.7, pseudo-t tests 2.59 to 4.77) and Mozambique districts, Cabo Delgado, Nampula and Zambezia in (range, $\mathrm{OR}=1.1$ to 1.2 , pseudo-t tests 2.59 to 4.77). Instead, in West Africa (Figure 6B), infants of women who reported not wanting or plan to have their last child were at very high odds of mortality in Niger's capital city Niamey $(\mathrm{OR}=2.1$, pseudo- $\mathrm{t}=7.32)$ and district Dosso $(\mathrm{OR}=3.1$, pseudo$\mathrm{t}=5.31)$ and Cascades $(\mathrm{OR}=1.1$, pseudo- $\mathrm{t}=4.96)$ Burkina Faso.

\section{Residual maps}

In East Africa, the final SGWRP model under-estimated neonatal mortality (Figure 7A) in the northern districts of Malawi, Zambezia in Mozambique and Mara and Lindi in Tanzania. In
West Africa, the final SGWRP model under-estimated neonatal mortality (Figure 7B) in the districts of Est in Burkina Faso and South East and South West, Nigeria. The residuals in both regions were not highly clustered indicating a good fit of the models.

\section{Discussion}

\section{Matching needs and place}

The purpose of this study was to identify where to target SDGinterventions to reduce neonatal mortality in the two highly prevalent regions of sub-regions of Africa, East Africa and West Africa. Three known dimensions of need were highlighted, including: i) where to improve care around the time of delivery, ii) where to increase mother's education, and iii) where to further empower women.

\section{East Africa}

While countries in East Africa had the lowest percentage of mothers who delivered at home (38.4\%, range, Mozambique $2.7 \%$ to Ethiopia 89.6\%); home birthing was a significant risk factor for neonatal mortality. Importantly, maternal exposure to unprotected water sources largely explained the home birth and neonatal mortality relationship, controlling for mothers with no education, mothers who did not want or plan to have their last child and rural residency. The findings from previous studies investigating mothers who deliver at home in East Africa may be summarised by rural residency (Stephenson et al., 2006; Ng'anjo Phiri, et al., 2014; Yebyo et al., 2015), pastoralist communities (Yebyo et al., 2015), communities in poverty (Yebyo et al., 2015), real or perceived travel distances to a health facility (Shiferaw et al., 2013; Sipsma et al., 2013; Yebo et al., 2015) and unreliable transportation ser-
A

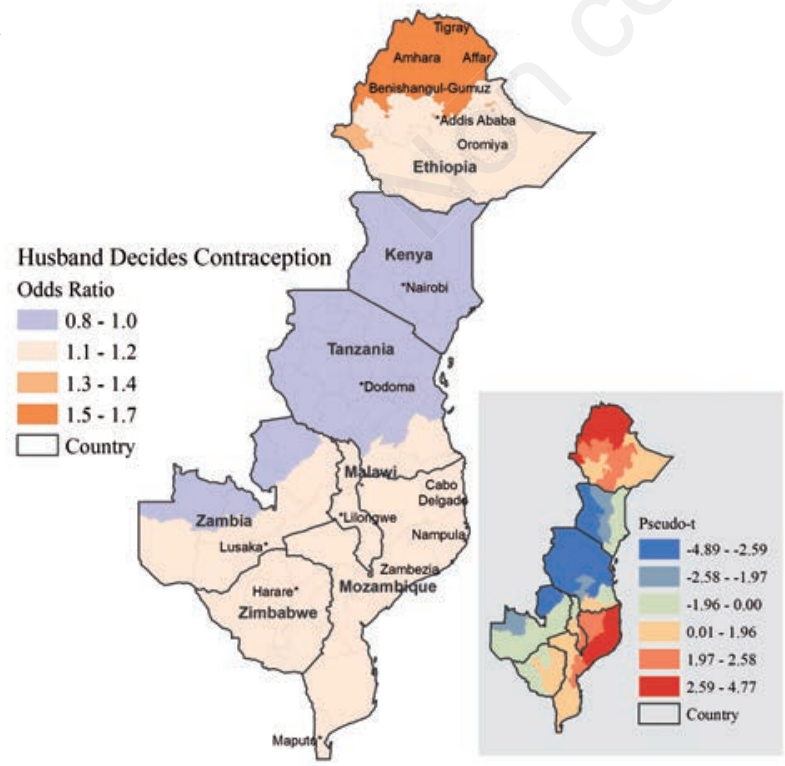

B

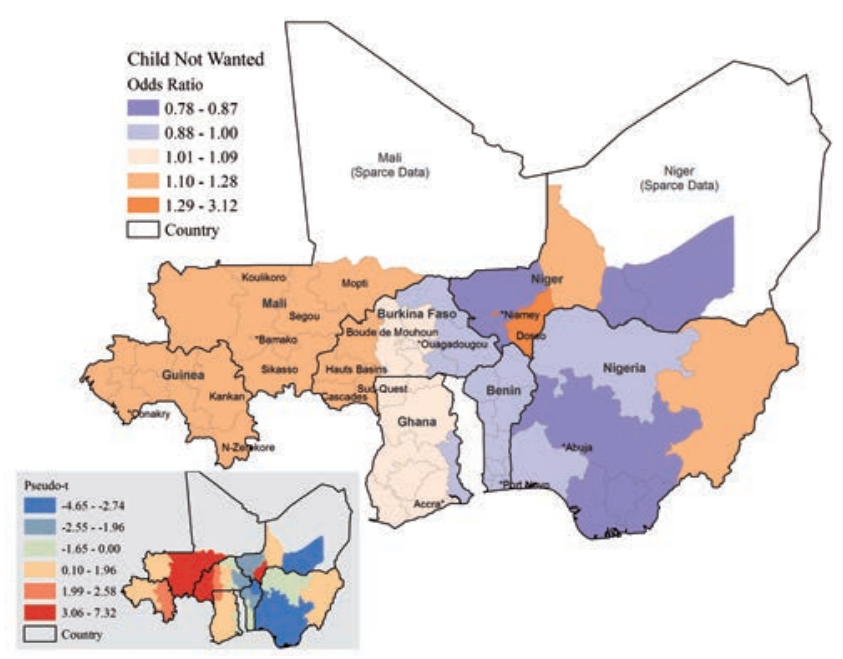

Figure 6. Estimated effect of women's empowerment dimension on neonatal mortality (controlling for variables, see Table 4), geographically weighted poisson regression analysis East Africa (A) and West Africa (B). Data were extracted from Birth Recode Data, the most recent survey year by country and region. Bivariate Global Moran's I: East Africa=0.193 and West Africa=-0.134. 
vices to a health facility (Shiferaw et al., 2013; Sipsma et al., 2013) especially during the rainy season (Kumbani et al., 2013). In addition, mothers who deliver at home may be characterised as those with fewer than 4 antenatal visits (Stephenson et al., 2006), those who are unable to pay for the high cost of maternity care (Stephenson et al., 2006; Shiferaw et al., 2013; Sipsma et al., 2013; Ng'anjo Phiri et al., 2014) and those who have concerns about the quality of maternity care (Sipsma et al., 2013), including health care worker's attitudes (Kumbani et al., 2013). Other factors may include religion - Protestant and Muslim in Tanzania, Protestant in Malawi (Johnson et al., 2013; Kumbani et al., 2013), husband's approval of family planning (Stephenson et al., 2006), the belief that it is not necessary or customary to attend a health care facility (Shiferaw et al., 2013) and pressure from husband or family members to deliver at home (Sipsma et al., 2013; Shiferaw 2013). Finally, mothers reported birthing at home due to rapid labor (Kumbani et al., 2013), onset of labor at night (Kumbani et al., 2013), low education and low exposure to media (Yebyo et al., 2015) and not wanting to go to a health care facility alone (Stephenson et al., 2006). There were mixed results in terms of the birth order and home delivery. Ybeyo et al. (2015) found that in Ethiopia, women with more children were more likely to deliver at home than women with fewer children. Stephenson et al. (2006) did not find differences between birth order and home births. In contrast, Johnson et al. (2013) found that in low and middle income countries, women with three and four or more births compared to women with one or two births were more likely to switch from home to a facility delivery. It is important to highlight that none of the above studies mentioned the complications of home

A

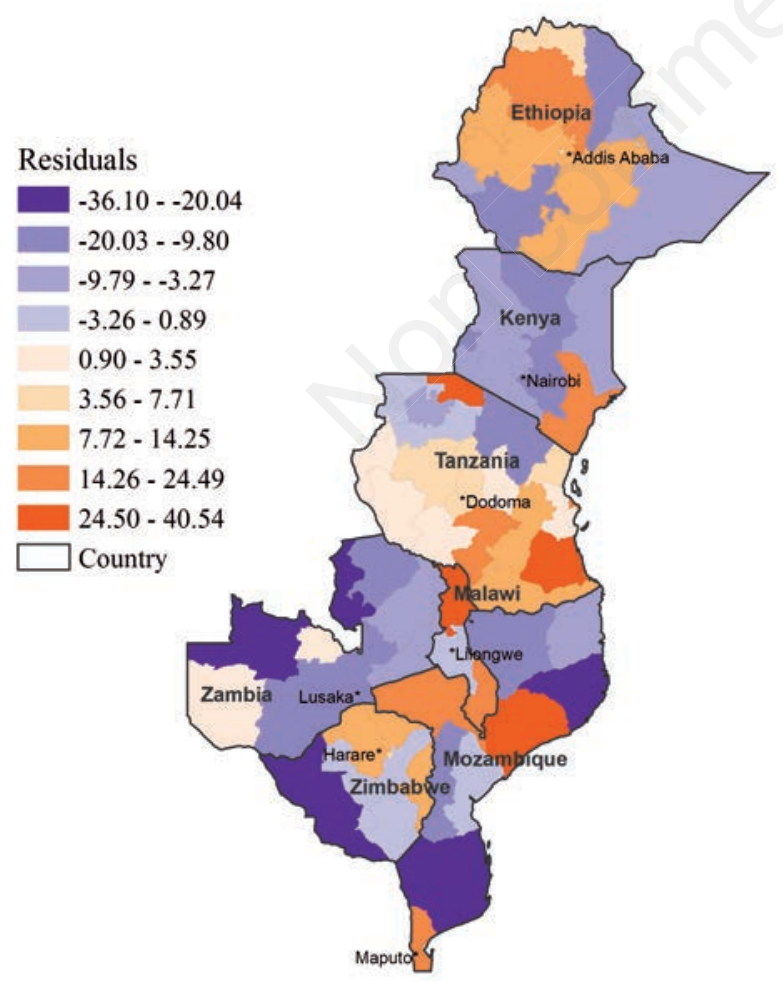

births associated with maternal exposure to unprotected water sources. A shortage of water or exposure to unclean water sources during or immediately after the home delivery may contribute to cord and/or intestinal infections, especially if the umbilical cord is not cleaned properly and/or the infant is fed a water-based diet instead of immediate breast feeding. In East Africa, 46.5\% of deliveries were not attended by a doctor or health professional. While studies have shown the challenges of water scarcity and hospital-acquired infection control (Adeyemo et al., 1993) and refugee situations (Toole and Waldman, 1997), there is a need to further investigate the contribution of water resources and home births mediated by care attendants to further reduce neonatal mortality in East Africa.

Finally, elevated neonatal mortality rates in East Africa, particularly in southern countries, were most significantly explained by women possessing no education. Furthermore, neonatal mortality influenced by empowerment of women was most significantly explained by mothers whose husband decided on their contraceptive practices. Studies have shown that if women are empowered and given control over everyday activities, such as household income, they are more likely to spend money on important things as good diet, health, education and childcare (Duflo, 2012). This has then led to the development of funds focused on empowering women in East Africa, namely East Africa Women's Economic Empowerment Program, 1992-2013 who specialise in giving grants and funds for Tanzania, Uganda, and Zimbabwe women (McKnight Foundation, 2013). Family planning is important in women's health. In Kenya, a new constitution was passed in August 2010, which advocated for free and voluntary family planning services for women, and for the first time, beginning in 20112012, the government will cover the full cost of contraceptive commodities (Population Reference Bureau, 2011). SDG-interventions that focus on the continued education of mothers in terms of

B

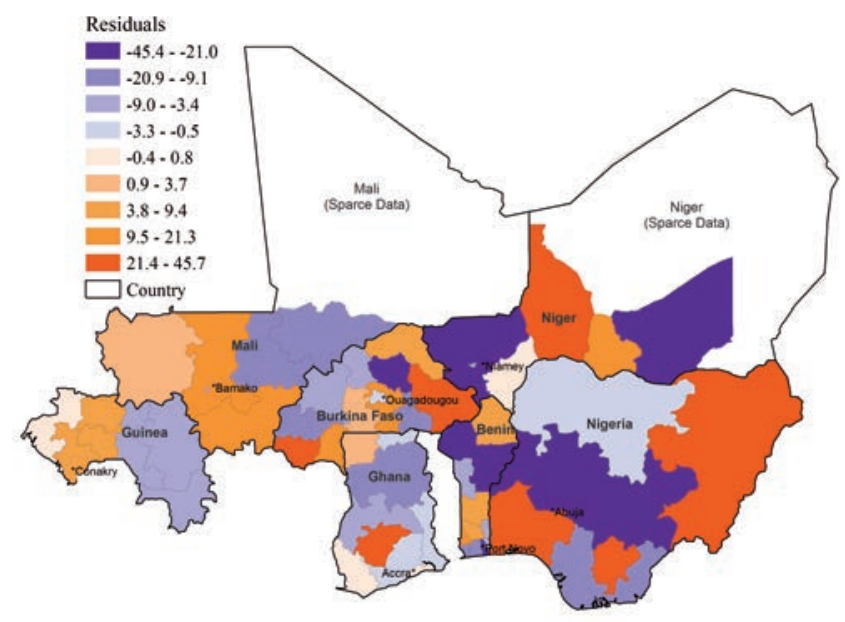

Figure 7. Residuals from modeled neonatal mortality (controlling for variables, see Table 4), geographically weighted Poisson regression analysis East Africa (A) and West Africa (B). Data were extracted from Birth Recode Data, the most recent survey year by country and region. Univariate Global Moran's I: East Africa $=0.018$ and West Africa=-0.071. 
schooling and also family-planning empowerment, particularly in Ethiopia and southern countries in East Africa, will be important strategies to further reduce neonatal mortality in that region.

\section{West Africa}

In West Africa, $48.6 \%$ of mothers delivered their infants at home (range, Benin $13.1 \%$ to Niger $70.3 \%$ ). Home delivery was a significant risk factor for neonatal mortality, particularly in eastern countries where the percentages of deliveries not attended by a doctor, midwife or health professional were also high. Importantly, mother's $\geq 35$ years of age and female infants largely explained the home birth and neonatal mortality relationship, controlling for mothers with primary education and mothers whose child was not wanted. The findings from previous studies investigating why mothers deliver at home in West Africa included rural residency (Johnson et al., 2009; Stephenson et al., 2006), savannah landscape which are relatively disadvantaged in terms of residential isolation, social development and access to available health care (Johnson et al., 2009), long travel distances to health facilities (Some et al., 2011), transportation difficulties (Some et al., 2011), especially with damaged roads from flooding during the rainy season (Some et al., 2011) and community poverty, (Some et al., 2011; Onubiwe Nkwo et al., 2014). Other explanations for mother's delivering at home in West Africa included less than 4 antenatal care visits (Johnson et al., 2009), high maternity care costs (Some et al., 2011), religion (Addai, 2003), if the husband's managed the household finances (Some et al., 2011) and family advised against facility birthing (Some et al., 2011). Mothers also delivered at home if the labor was fast and easy (Some et al., 2011), if they were young (15-19 years) (Onubiewe Nkwo et al., 2014) or middle aged, 30-49 years in Burkina Faso (Stephenson et $a l ., 2006)$, if they had less than a secondary education (Johnson et al., 2009) or if the birth interval was shorter than 8 months (Onubiewe Nkwo et al., 2014). In northern Nigeria, mother's attending antenatal care and HIV related counselling and testing services (Ng'anjo Phiri et al., 2014) and exposed to family planning information (Stephenson et al., 2006) were more likely to delivery at a health facility. Relatedly, in Burkina Faso and Ghana mothers were more likely to deliver in a hospital or health care facility if the mother received family planning information or had a previous birth in a hospital (Stephenson et al., 2006). In Guinea and Nigeria, mothers who had three or more births were more likely to deliver at a health facility than mothers whose birth was their first or second (Johnson et al., 2009).

In this study, the association between home birth and neonatal mortality in West Africa was largely explained by maternal age $\geq 35$ years and female infants, controlling for other significant dimensions of need at the district level. Older mothers may resist attending health facilities to deliver if the facility is perceived as too modern. Complications associated with advanced maternal age included a higher likelihood of developing gestational diabetes and/or hypertension, birthing a low-birth weight $(<2500 \mathrm{~g})$ infant, infant having chromosome abnormalities, or pregnancy loss either by miscarriage or stillbirth (American Congress of Obstetrics and Gynecology, 2015). Furthermore, neonatal mortality was significantly explained by mothers who did not want or plan to have their last child. Given the trend toward women having children later in life (Pew Research Center, 2010), target interventions in West Africa should emphasise improvements in prenatal care, including family planning education, and increased training and specialisation of birth attendants to manage and encourage facility deliveries for women of advanced maternal age.

Finally, prior research has demonstrated a distinct bias against female children throughout several nations across the world (D'Souza and Chen, 1980; Coale and Banister, 1994; Park and Cho, 1995). Addressing culturally-sensitive biases toward infant girls in West Africa will be an important area of future SDG-intervention.

The findings from this study demonstrate the need to model the spatially varying dimensions of need to target specific districts and countries, to reduce the burden of neonatal mortality in East Africa and West Africa. There are however, a few limitations to this study. First, this study included differences in the years of the DHS datasets ranging from East Africa 2008 to 2014 and West Africa 2010 to 2014 , which may lead to district, country and regional biases associated with medical advances and changes in programmatic initiatives that address neonatal mortality during those time periods. This study also did not assess the causes of neonatal mortality and how they differed by district, country and region in response to these changes across the time periods. The definition of home delivery could include the mother's home or another home, which may not be comparable. Successive births and previous births that lead to mortality were also not assessed to see if mother's changed their birthing location over time. In addition, trained birth attendants have been shown to be of little help to women who develop complications at birth (Rosenfield et al., 2007; Costello et al., 2006) a phenomenon that could not be measured in this study. In West Africa, mothers who did not want their last child could mean that they wanted the child later or they wanted the child no more which could result in different interpretations. More information on family planning services would have also helped in the overall interpretation of mother's desire for pregnancy and home birth $v s$ facility deliveries. Furthermore, this study investigated known dimensions of need independently; however, these needs may also have operated interactively or along different pathways. For example, attendants of mothers who delivered at home may be someone other than a doctor, midwife or health professional or a mother who did not want her child may also have had a husband who decided on their contraception practice. Finally, this study did not address the potential of social, cultural and incidence biases associated with including Malawi, Mozambique and Zimbabwe - countries with widespread regional designation in Southern Africa in the East Africa regional analysis.

\section{Final considerations}

Interventions should begin by considering the current barriers to women attaining an education, despite a growing emphasis in widening access to education (Goal 4). In East Africa, mothers with no education were a significant risk factor for neonatal mortality; however, in West Africa, primary education was also a significant risk factor for neonatal death. Ensuring that women stay in school and acquire at least a secondary education (Goal 4.3) that include technical and vocational skills for employment will also be important interventions to reduce neonatal mortality. Finally, this study demonstrated the broad need to further reduce gender inequality (Goal 5) by ensuring the survival of female infants in West Africa and the empowerment of women to make decisions on her contraceptive practices, particularly in East Africa that would also reduce the number of unwanted pregnancies in West Africa. Although the justification of wife-beating was not a significant risk factor for neonatal mortality in this study, the district percentages of women with husbands who have this belief is high across both 
regions. Empowerment should also therefore, include the elimination of violence against women and girls (Goals 5.1,2), ensure universal access to sexual and reproductive health rights (Goal 5.6) and adopt sound policies and enforceable legislation (Goal 5.9) to reduce domestic violence, prevent trauma and chronic diseases in mothers and miscarriages and premature birth in pregnancy. This health geographic study highlighted districts within countries in the highest neonatal mortality regions, where SDG-interventions as outlined above can be prioritised and implemented.

\section{Conclusions}

This study recommends that future SDG-interventions pertaining to neonatal mortality be incorporated into health and nonhealth goals in districts and countries in East Africa and West Africa. For mothers who delivered at home, health care financing should include the continued training of health professionals, including midwives and relatives who can recognise and rapidly address delivery complications, including asphyxia, infections and the need for child warmth and breastfeeding immediately after delivery. In addition, the structural and cultural barriers to delivering at a health care facility should be examined. These concerns may be addressed in Goal 3.87 to creating resilient maternity health care systems that are well governed and financed, including universal access to sexual and reproductive health-care services. Within resilient maternity health care systems there should also be a focus on capacity-building to ensure the availability of safe and affordable clean water and sanitation to ensure hygienic delivery conditions and protected water intake for mothers and infants within health facilities both at home and in their communities (Goal 6).

\section{References}

Addai I, 2003. Determinants of use of maternal-child health services in rural Ghana. J Biosoc Sci 32:1-15.

Adeyemo AA, Akiindele JA, Omokhodion SL, 1993. Klebsiella septicaemia, osteomyelitis and septic arthritis in neonates in Ibadan, Nigeria. Ann Trop Paediatr 13:285-9.

American Congress of Obstetrics and Gynecology, 2015. Having a baby after age 35. Available from: http://www.acog.org /Patients/FAQs/Having-a-Baby-After-Age-35

Anselin L, Rey S, 1991. Properties and tests for spatial dependence in linear regression models. Geogr Anal 23:112-31.

Bicego GT, Boerma JT, 1993. Maternal education and child survival: a comparative analysis. Soc Sci Med 36:1207-27.

Caldwell JC, 1979. Education as a factor in mortality decline: an examination of Nigerian data. Pop Stud 33:395-413.

Coale AJ, Banister J, 1994. Five decades of missing females in China. Demography 31:459-79.

Costello A, Azad K, Barenette S, 2006. An alternative strategy to reduce maternal mortality. Lancet 368:2122-3.

D'Souza S, Chen LC, 1980. Sex differential in mortality in rural Bangladesh. Pop Develop Rev 6:257-70.

Duflo E, 2012. Women empowerment and economic development. J Econ Lit 50:1051-79.

Engmann C, Walega P, Aborigo RA, Adongo P, Moyer CA, Lavansani L, Williams J, Bose C, Binka F, Hodgson A, 2012. Stillbirths and early neonatal mortality in rural Northern
Ghana. Trop Med Int Health 17:272-82.

ESRI, 2015. Environmental Systems Research Institute. Available from: http://www.esri.com

Feuillet T, Charreire H, Menai M, Salze P, Simon C, Dugas J, Oppert J-M, 2015. Int J Health Geogr 14:12.

Fotheringham AS, Brunsdon C, Charlton M, 2002. Geographically weighted regression the analysis of spatially varying relationships. John Wiley \& Sons Ltd., New York, NY, USA.

Hamilton BE, Martin JA, Ventura SJ, 2006. Births: preliminary data for 2005. National vital statistics reports 55;11. National Center for Health Statistics, Hyattsville, MD, USA.

Hobcraft J, 1993. Women's education, child welfare and child survival: a review of the evidence. Health Transit Rev 3:159-75.

Johnson FA, Padmadas SS, Brown JJ, 2009. On the spatial inequalities of Institutional versus home births in Ghana: a multilevel analysis. J Commun Health 34:64-72.

Johnson FA, Padmadas SS, Matthews Z, 2013. Are women deciding against home births in low and middle income countries? PLoS One 8:e65527.

Kumbani L, Bjune G, Chirwa E, Malata A, Odland JØ, 2013. Why some women ail to give birth at health facilities: a qualitative study of women's perceptions of perinatal care from rural Southern Malawi. Reprod Health 10:9.

Lawn JE, Manandhar A, Haws RA, Darmstadt GL, 2007. Reducing one million child deaths from birth asphyxia: a survey of health systems gaps and priorities. Health Research Policy Syst 5:4.

Lawn JE, Osrin D, Alder A, Cousens S, 2008. Four million neonatal deaths: counting and attribution of cause of death. Paediatr Perinat Epidemiol 22:410-6.

McKnight Foundation, 2013. East Africa women's economic empowerment program, 1992-2013 report. McKnight Foundation, Minneapolis, MN, USA.

Merck Manual, 2015. Available from: http://www.merckmanuals. $\mathrm{com} /$ professional/SearchResults?query=premataure+labor

Ng'anjo Phiri S, Kiserud T, Kvåle G, Byskov J, Evjen-Olsen B, Michelo C, Echoka E, Fylkesnes K, 2014. Factors associated with health facility childbirth in districts in Kenya, Tanzania and Zambia: a population based survey. BMC Pregn Child 14:219.

Onubiwe Nkwo P, Lawani LW, Ezugwu EC, Iyoke CA, Ubesie AC, Onoh RC, 2014. Correlates of poor perinatal outcomes in non-hospital births in the context of weak health system: the Nigerian experience. BMC Pregn Child 14:341.

Park CB, Cho N-H, 1995. Consequences of son preferences in a low fertility society: imbalance of the sex ratio at birth in Korea. Pop Develop Rev 21:59-84.

Pew Research Center, 2010. Older mothers. Available from: http://www.pewresearch.org/daily-number/older-mothers/

Population Reference Bureau, 2011. Kenya population data sheet. Population Reference Bureau and National Coordinating Agency for Population and Development, Washington, DC, USA.

Rosenfield A, Min CJ, Freedman LP, 2007. Making motherhood safe in developing counties. New Engl J Med 356:1395-7.

Rutstein SO, Rojas G, 2006. Guide to demographic and health survey (DHS) statistics. Demographic and health surveys methodology. ORC Macro, Calverton, MD, USA.

Shiferaw S, Spigt M, Godefrooij M, Melkamu Y, Tekie M, 2013. Why do women prefer home births in Ethiopia? BMC Pregn Child 13:5. 
Sipsma H, Thompson J, Maurer L, Bradley E, Curry L, 2013. Preferences for home delivery in Ethiopia: provider perspectives. Glob Public Health 8:1014-6.

Some TD, Sombie I, Meda N, 2011. Women's perceptions of homebirths in two rural medical districts in Burkina Faso: a qualitative study. Reprod Health 8:3.

Stephenson R, Baschieri A, Clements S, Hennink M, Madise N, 2006. Contextual influences on the use of health facilities for childbirth in Africa. Am J Public Health 96:84-93.

Toole MJ, Waldman RJ, 1997. The public health aspects of complex emergencies and refugee situations. Annu Rev Public Health 18:283-312.

UN, 2015. United Nations Millennium Development Goals (UN MDGs) Report. Available from: http://www.undp.org/content/dam/undp/library/MDG/english/UNDP_MDG_Report_2 015.pdf

UN, 2016. United Nations Sustainable Development Goals (UN SDGs). Available from: https://sustainabledevelopment. un.org/

UNICEF, 2015. Levels and trends in Child mortality Report. United Nations Inter-agency group for child mortality estima- tion 29. United Nations Children's Fund, New York, NY, USA.

Wheeler D, Tiefelsdorf M, 2005. Multicollinearity and correlation among local regression coefficients in geographically weighted regression. J Geogr Syst 7:161-87.

WHO, 2004. The global burden of disease. World Health Organization, Geneva, Switzerland. Available from: http://www.who.int/healthinfo/global_burden_disease/2004_r eport_update/en/

WHO, 2015a. Data neonatal mortality. Global Health Observatory, World Health Organization, Geneva, Switzerland. Available from: http://www.who.int/gho/child_health/mortality/neonatal/en

WHO, 2015b. From MDGs millennium development goals to SDGs sustainable development goals. World Health Organization, Geneva, Switzerland. Available from: http://apps.who.int/iris/bitstream/10665/200009/1/978924156 5110_eng.pdf?ua $=1$

Yebyo H, Alemayehu M, Kahsay A, 2015. Why do women deliver at home? multilevel modeling of Ethiopian national demographic and health survey data. PLoS One 10:e0124718. 